\title{
Etching Method to Create Random Speckle Pattern on Semi-Solid Cast A356 Aluminium for DIC in-situ strain measurement
}

\author{
Maëlle Sergolle ${ }^{\mathrm{a}}$, Jorge Santos ${ }^{\mathrm{b}}$, Keivan A. Kasvayee ${ }^{\mathrm{b}}$, Anders E.W. Jarfors*b \\ ${ }^{a}$ Ecole Nationale Supérieure d'Ingénieurs de Caen - ENSICAEN, 6 boulevard Maréchal Juin, CS 45053, 14050 Caen cedex 04, France \\ 'School of Engineering, Jönköping University, Box 1026, 55111 Jönköping, Sweden \\ *e-mail: anders.jarfors@ju.se
}

Received: 15 December 2016/Accepted: 17 January 2017/Published online: 17 March 2017

This article is published with open access at AGH University of Science and Technology Press

\begin{abstract}
Aluminum semi-solid casting is constantly evolving, as it offers a combination of reduced shrinkage porosity and gas entrapment defects together with high productivity and an extended die-life. The relationship between the microstructure and stress-strain behavior is not well-understood due to its non-conventional microstructure. In-situ tensile testing, combined with optical microscope and Digital Image Correlation (DIC), has been used for local strain distribution measuements in cast irons. The critical capability was an etching technique to generate a micro-scale random speckle pattern with a sufficiently high speckle density to enable the sufficient spatial resolution of displacement and strain. The current paper focuses on the development of a pit etching procedure for the semi-solid cast A356 aluminum alloy to study local strain accommodation on the microstructure during tensile loading. The critical challenge of this procedure was the generation of homogeneously distributed pits on both the primary aluminum and eutectic regions. Therefore, a heated solution used for wet-etch aluminum in microfabrication was modified as well as a process adapted to generate pits with suitable characteristics. In-situ tensile tests were performed attached to an optical microscope to record the microstructure and displacements during loading. DIC software was used for analysis. The procedure was validated through a comparison between the resulting Young's moduls using standard tensile testing and the DIC process on the speckle pattern generated. A good fit between the two methods for Young's modulus was found. The spatial resolution obtained was, however, not sufficient to fully resolve the strain gradients in the microstructure, but it did reveal large strain variations in the microstructure.
\end{abstract}

Keywords:

random speckle pattern, cast semi-solid aluminum alloy, pit etching, pitting corrosion, Digital Image Correlation (DIC), local strain

\section{INTRODUCTION}

Semi-Solid Metal (SSM) casting has been used in production for some time now and has proven to be capable of producing high-density sound components. The main process step is the injection of a slurry into a die cavity by applying pressure. SSM processing is performed at a lower temperature than liquid casting as compared to conventional High Pressure Die Casting (HPDC). The lower temperature and heat input associated with SSM processing decrease the thermal load on the die and help increase die life. Moreover, shrinkage porosity is lessened due to the injection of a partially solidified material, gas entrapment porosity is reduced as viscosity is increased, and conditions for laminar flow during die filling are improved [1, 2].

The goal of this paper is to develop a speckle patterning technique for Digital Image Correlation (DIC) analysis of local strains in a rheocast aluminum at a microscopic scale during an in-situ tensile test. Similar work was successfully developed for ferritic castiron, enabling measurements of local strain distributions in the microstructure of cast iron. DIC is an effective tool for the determination of complex strain using full-field methods. To perform DIC, a random speckle pattern must be produced on the sample surface to allow displacement measurements. For ferritic cast iron, a pit-etching technique was used to make it in order to increase the spatial resolution of DIC measurements [3]. This technique keeps the microstructure visible. The speckle pattern must meet certain criteria to allow for good DIC measurement. First, it must be a random pattern. Second, it must adhere to the surface and move along with it during deformation. The pattern also has to show a high speckle density and good contrast to limit errors. It must also be of suitable size, which means it must not be too big, cover an entire grain (for micro-scale level analysis), nor smaller than $3 \times 3$ pixels to allow matching in the full field of view [4]. 
When the pattern matches the requirements, the in-situ tensile test can be performed along with pictures recorded by the optical microscope software. To finish, they are analyzed with DIC to evaluate different properties [5, 6].

The following work aims to develop an etching technique to enable the study of local strain distribution in Semi-Solid Metal (SSM) A356 (AlSi7Mg0.3) cast material. The main challenge is to develop a way to make an appropriate random speckle pattern on the sample surface and to keep the microstructure visible. Compared to the previous work on cast iron [3], the idea is to cause pitting corrosion defined by the local perforations of the passive oxide layer mainly due to the presence of chloride or cupric ions in the environment. The A356 alloy is known for its corrosion resistance [7, 8]. However, several studied have shown that aluminum can be corroded with the presence of chloride ions (which are small and mobile) [9-12], even in the $\mathrm{pH}$ range where the alumina oxide layer is stable [13].

\section{EXPERIMENTAL PROCEDURE}

\subsection{Material and samples}

The material used for this work was a hypoeutectic aluminum alloy A356 containing around 7\% silicon, $0.3 \%$ magnesium, and iron limited to $0.14 \%$ (AlSi7Mg0.3). The slurry preparation method was Rheocasting ${ }^{\mathrm{TM}}$ [14]. After slurry preparation, the first sample (Fig. 1a) was cast in a 50-ton Vertical High Pressure Die Casting (VHPDC) machine. From the non-useful part of this piece, standard flat samples were cut (Fig. 1d). From the midsection, non-standard tensile samples (Fig. 1c) were produced by milling.

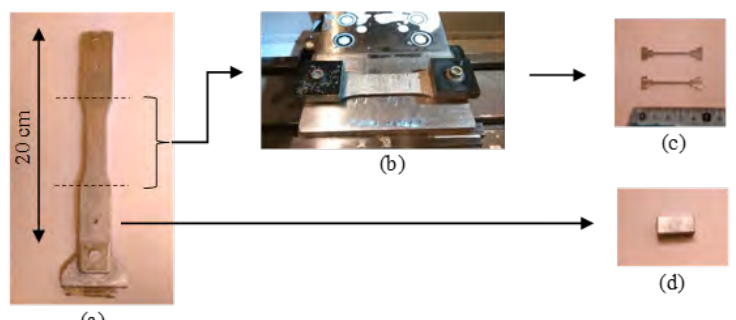

Fig. 1. Machining of samples: (a) cast sample; (b) machining in the CNC; (c) final tensile samples; (d) standard flat sample

The standard flat samples were used to develop the pit etchingtechnique to beapplied on thenon-standard tensile samples. Each sample was mounted and metallographically prepared before pit etching. Subsequently, the resin was removed to demount the test specimen before in-situ tensile testing. The final as-cast microstructure is shown in Figure 2.

The microstructure was typical for an SSM-processed material with primary $\alpha$-Al particles formed during slurry production (primary $\alpha$-Al) and finer scale secondary precipitated $\alpha$-Al formed during solidification in the die cavity (secondary $\alpha$-Al) as well as an eutectic consisting of $\alpha-\mathrm{Al}$ and Si particles.

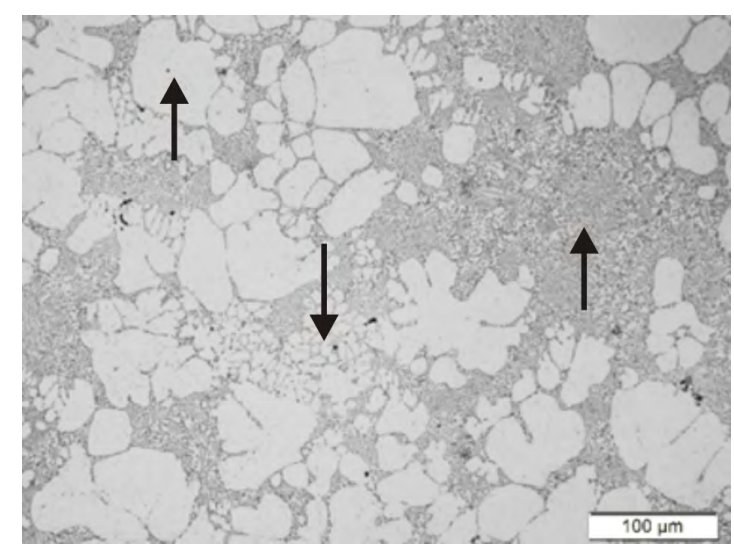

Fig. 2. Microstructure of SSM cast A356 revealed after $10 \mathrm{~s}$ in $\mathrm{NaOH} 10 \%$, where left arrow indicates primary particle of $\alpha-\mathrm{Al}$, middle arrow indicates secondary particle of $\alpha-\mathrm{Al}$, and right arrow indicates $\alpha$-Al-Si eutectic

\subsection{Pit etching}

In order to produce pits on both the $\alpha$-Al and eutectic regions, the solution of Wolf and Tauber [15] was modified. One part of hydrochloric acid was added to this solution, partially replacing phosphoric acid. Therefore, a reagent containing $\mathrm{H}_{3} \mathrm{PO}_{4}, \mathrm{CH}_{3} \mathrm{COOH}, \mathrm{HNO}_{3}$, $\mathrm{HCl}$, and $\mathrm{H}_{2} \mathrm{O}$ was used to produce the pattern. The tested solutions are given in Table 1 . Moreover, Solution A was tested at room temperature, $40^{\circ}, 55^{\circ}, 70^{\circ}$, and $85^{\circ} \mathrm{C}$ for optimization purposes.

Table 1

Chemical composition (vol.\%) of solutions used on standard flat samples

\begin{tabular}{cccccccc}
\hline Solution & $\mathbf{A}$ & $\mathbf{1}$ & $\mathbf{2}$ & $\mathbf{3}$ & $\mathbf{4}$ & $\mathbf{5}$ & $\mathbf{6}$ \\
\hline $\mathrm{H}_{3} \mathrm{PO}_{4}$ & 70 & 70 & 70 & 75 & 70 & 80 & 40 \\
\hline $\mathrm{CH}_{3} \mathrm{COOH}$ & 5 & 5 & 10 & 0 & 5 & 5 & 5 \\
\hline $\mathrm{HNO}_{3}$ & 5 & 5 & 5 & 5 & 10 & 5 & 5 \\
\hline $\mathrm{HCl}$ & 10 & 15 & 10 & 10 & 10 & 10 & 10 \\
\hline $\mathrm{H}_{2} \mathrm{O}$ & 10 & 5 & 5 & 10 & 5 & 0 & 40 \\
\hline
\end{tabular}

The procedure shown in Figure 3 was followed for reagent application on the standard flat samples. To finish, different implementation procedures were tested on standard flat and tensile samples.

From the pictures recorded, image-analysis software was used to calculate pit concentration, mean area, mean radius, and average distance from the nearest neighbors.

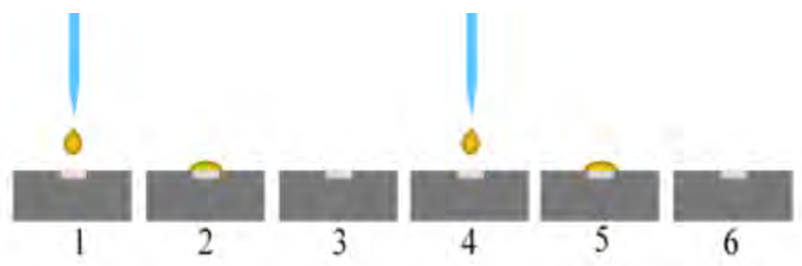

Fig. 3. Steps of pit etching implementation - 1 and 4); apply a drop - 2 and 5); wait 5 s-3 and 6); clean (water and ethanol) and dry 
The mean radius was given by the radius of a circle with the same area as the object. The nearest neighbor distance (NND) was calculated by the shortest distance from the center of gravity of each pit to another pits center of gravity. It is necessary to obtain at least $3 \times 3$ pixels patterns to allow the use of DIC $[3,4,16]$; hence, only pits with a minimum area of $2.69 \mu \mathrm{m}^{2}$ were selected (area of $3 \times 3$ pixels in $63 \mathrm{X}$ magnification).

\subsection{Tensile testing for procedure validation}

In order to analyze the pattern produced by pit etching and follow it during the in-situ tensile test, an invert optical microscope (Olympus GX81) equipped with a CCD camera (Olympus UC30) was used.

The in-situ tensile equipment (TSL Solutions KK, Japan) allows for the application of a dual-direction uniaxial tensile load with a maximum load capacity of $1200 \mathrm{~N}$. In order to follow the displacement and calculate local strains, this machine was installed on the inverted optical microscope (Fig. 4a). Figure 4b shows the dimensions of the tensile sample used for the in-situ tensile machine. Tensile testswere performed untilrupture with a crosshead speed of $3.3 \mu \mathrm{m} / \mathrm{s}$. A displacement calibration was made with an extensometer and a near-rigid steel sample to calibrate the machine's compliance.

Images were recorded at $63 \mathrm{X}$ magnification in grayscale every 3 seconds utilizing the autofocus function. The in-situ device recorded the overall load and displacement every 0.5 seconds. Stress-strain curves could be

a)

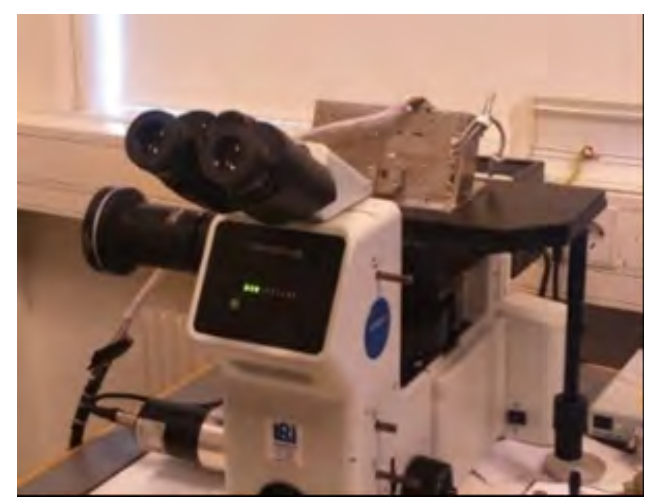

b)

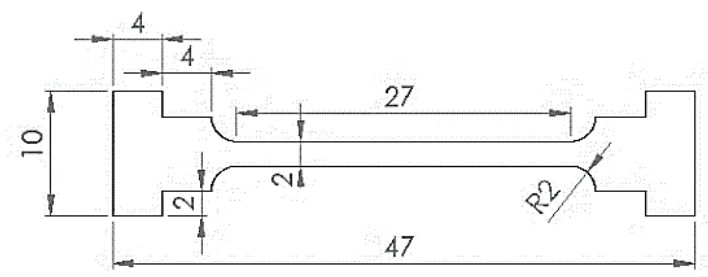

Fig. 4. In-situ tensile machine installed on the optical microscope (a); sample geometry adopted for the machine [6], with around $1.3 \mathrm{~mm}$ thickness (b) deduced from the initial data and compared with curves obtained with a standard tensile test; Young's modulus was used for validation.

\subsection{Digital Image Correlation}

The commercial MatchID 2D software was used to assess strain distribution. It is subset-based and capable of providing full-field displacement and strain data on planar specimens. This program was adapted to the interpretation of results in a quantitative way with integrated error assessment [17]. Stationary pictures were analyzed, and an appropriate subset size was determined according to the pattern produced. Moreover, displacement and strain spatial resolution were calculated. Then, local strain measurements could be done on a set of deformed images recorded during the in-situ tensile test. The elastic modulus was calculated using the strain fields from the recorded images of the deformed sample in the elastic regime. This was chosen as a critical test case as small strain measurements were the most challenging conditions from noise and resolution standpoints.

\section{RESULTS AND DISCUSSION}

\subsection{Speckle pattern}

Figure 5 shows the microstructure of standard flat samples before and after pit etching with Solution A (Tab. 1).

a)

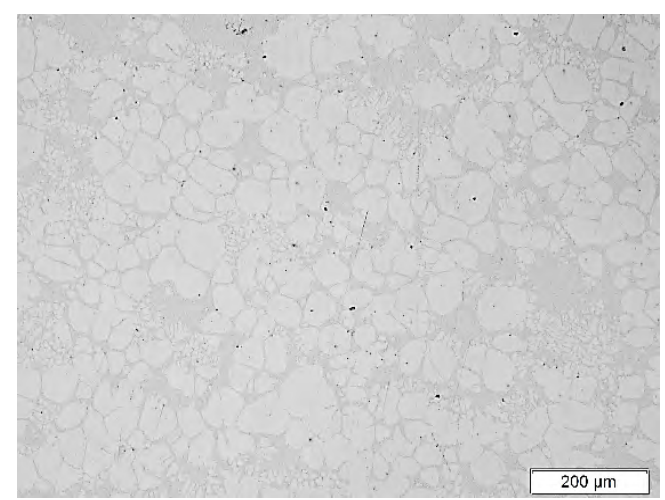

b)

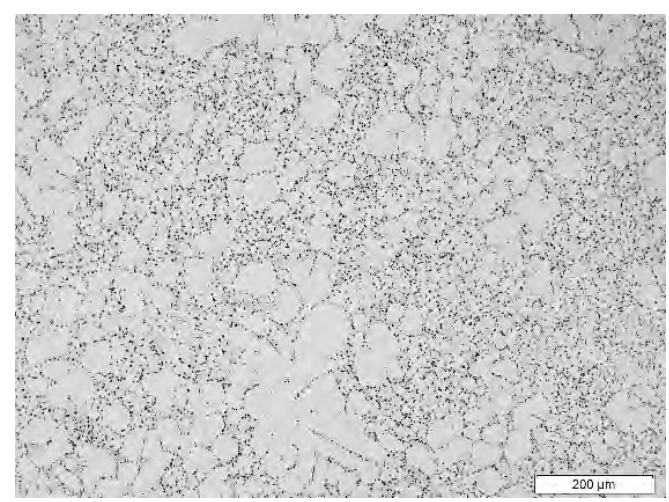

Fig. 5. Standard flat sample microstructures: (a) un-etched; (b) etched with reagent $A$ 
Table 2 presents the size and distribution of the speckles for each reagent concentration. The increase of chloride ion concentration tended to generate larger pits, with lower spatial homogeneity (Solution 1) as compared to Solution A. An inappropriate quantity of acetic acid resulted in low pit density (Solutions 2 and 3). The addition of nitric acid produced too-large pits (Solution 4) primarily driven by reactivity in the eutectic regions. To finish, an appropriate proportion of water was needed to obtain a better pattern. Without water, Solution 5 did not generate a sufficiently high density of pits. However, Solution 6 (with the largest amount of water) almost completely inhibited the formation of pits.

Wolf and Tauber [15] stated that, for microfabrication with wet etching of silicon and aluminum, nitric acid is able to form aluminum oxide on the surface. Phosphoric acid and water dissolve this material simultaneously. Moreover, in this study, the high viscosity of $\mathrm{H}_{3} \mathrm{PO}_{4}$ may decrease reactivity in the eutectic region, as the mobility of species is reduced. The addition of acetic acid may reduce the dissociation of $\mathrm{HNO}_{3}$ and, thus, improve the effect of this oxidation agent. In order to pit etch the aluminum phases, chloride ions are essential.

These results substantiated the use of Reagent $\mathrm{A}$ with 70\% phosphoric acid, 5\% acetic acid, 5\% nitric acid, $10 \%$ hydrochloric acid, and $10 \%$ water for pit etching of the semi-solid cast alloy A356. These results substantiated the use of Reagent A with $70 \%$ phosphoric acid, 5\% acetic acid, 5\% nitric acid, 10\% hydrochloric acid, and $10 \%$ water for pit etching of the semi-solid cast alloy A356. However, by etching standard flat samples, the primary $\alpha$-Al was not sufficiently etched. In an attempt to optimize the procedure, the temperature was varied to increase the reactivity of the primary $\alpha$-Al phase. Table 3 shows the size and distribution of the speckles for Solution A used at various temperatures. The use of $70^{\circ} \mathrm{C}$ allowed for a significant increase in pit density and a necessary reduction of the mean distance between pits. However, above this critical temperature, there was a decline in pit density. At $20^{\circ} \mathrm{C}$, it was obvious that the number of pits was insufficient. At $70^{\circ} \mathrm{C}$, the nearest-neighbor-distance distribution was the most homogeneous. Thus, the optimum condition was to use Solution A at $70^{\circ} \mathrm{C}$.

Because of the difficulty of generating pits in the primary $\alpha-\mathrm{Al}$, the implementation of pit etching was changed. For this, each etching procedure was tested on both types of samples (standard flat and tensile samples; see Figure 1). The reagent was, thus, applied differently: (a) sample directly immeged for $10 \mathrm{~s}$ in the agitated solution; (b) a drop of the solution was deposed on the sample surface for $10 \mathrm{~s}$; (c) a drop of the solution was deposed on the sample surface for $5 \mathrm{~s}$; the sample was cleaned with water and ethanol then dried; another drop was deposed for 5 s (see Figure 3).

Figure 6 shows the difference between the pit etching of the standard flat and the non-standard tensile samples.

a)

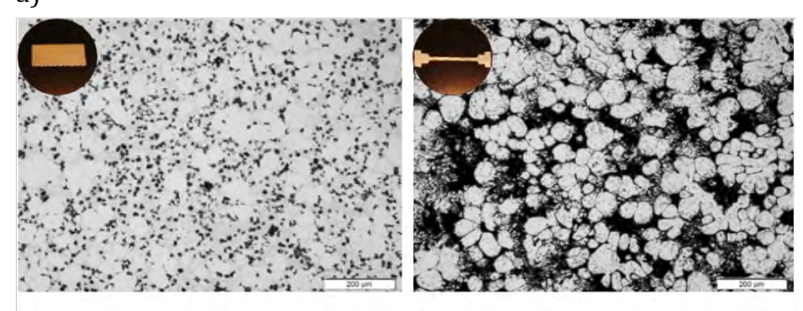

b)

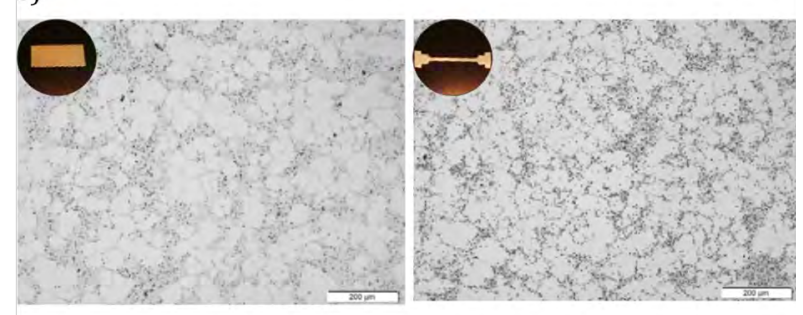

c)

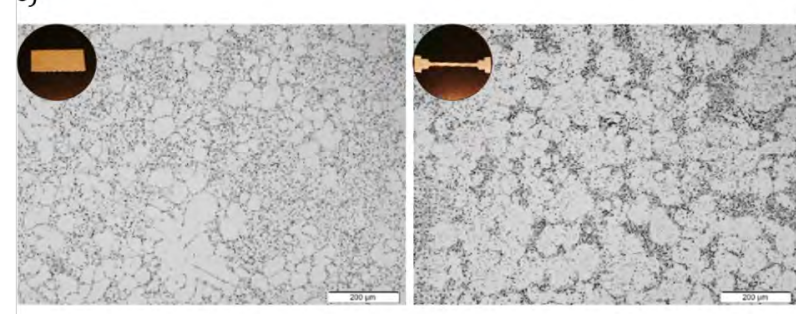

Fig. 6. Microstructure of A356 in standard flat (left) and tensile samples (right) pit etched with Reagent $\mathrm{A}$ at $70^{\circ} \mathrm{C}$ with three procedures: a) method $\mathrm{A}$; b) method $\mathrm{B}$; and $\mathrm{c}$ ) method $\mathrm{C}$

Table 2

Size and distribution of the speckles calculated for each pattern according to reagent concentration

\begin{tabular}{cccccccc}
\hline Solution & $\mathbf{A}$ & $\mathbf{1}$ & $\mathbf{2}$ & $\mathbf{3}$ & $\mathbf{4}$ & $\mathbf{5}$ & $\mathbf{6}$ \\
\hline Number density $\left(1 / \mathrm{mm}^{2}\right)$ & 6586 & 5201 & 3868 & 3924 & 4194 & 4343 & 83 \\
\hline Average area $\left(\mu \mathrm{m}^{2}\right)$ & $7.3 \pm 4.6$ & $9.6 \pm 7.9$ & $7.0 \pm 4.0$ & $6.8 \pm 4.6$ & $13.2 \pm 11.2$ & $8.0 \pm 4.5$ & $5.1 \pm 3.1$ \\
\hline Mean radius $(\mu \mathrm{m})$ & $1.5 \pm 0.4$ & $1.7 \pm 0.6$ & $1.4 \pm 0.4$ & $1.4 \pm 0.4$ & $1.9 \pm 0.8$ & $1.5 \pm 0.4$ & $1.2 \pm 0.3$ \\
\hline Mean NND $(\mu \mathrm{m})$ & $5.5 \pm 2.1$ & $6.3 \pm 2.5$ & $6.7 \pm 2.9$ & $6.4 \pm 2.9$ & $6.6 \pm 2.9$ & $6.1 \pm 2.6$ & $23.6 \pm 15.5$ \\
\hline
\end{tabular}

Table 3

Size and distribution of speckles calculated for each pattern according to solution temperature

\begin{tabular}{cccccc}
\hline Temperature & $\mathbf{2 0}{ }^{\circ} \mathbf{C}$ & $\mathbf{4 0}^{\circ} \mathbf{C}$ & $\mathbf{5 5}^{\circ} \mathbf{C}$ & $\mathbf{7 0}^{\circ} \mathbf{C}$ & $\mathbf{8 5}^{\circ} \mathbf{C}$ \\
\hline Number density $\left(1 / \mathrm{mm}^{2}\right)$ & 563 & 1809 & 1905 & 6586 & 2013 \\
\hline Average area $\left(\mu \mathrm{m}^{2}\right)$ & $4.9 \pm 2.3$ & $9.1 \pm 7.9$ & $7.8 \pm 6.0$ & $7.3 \pm 4.6$ & $7.4 \pm 5.3$ \\
\hline Mean radius $(\mu \mathrm{m})$ & $1.2 \pm 0.3$ & $1.6 \pm 0.6$ & $1.5 \pm 0.5$ & $1.5 \pm 0.4$ & $1.5 \pm 0.5$ \\
\hline Mean NND $(\mu \mathrm{m})$ & $11.1 \pm 6.6$ & $8.0 \pm 4.6$ & $8.6 \pm 4.5$ & $5.5 \pm 2.1$ & $8.1 \pm 4.4$ \\
\hline
\end{tabular}


It was easier to etch tensile samples (more sensitive to corrosion), and some pits could be observed in the primary $\alpha$-Al particles. However, there were always more pits in the eutectic regions than in any of the aluminum particles. This could be due to the difference of potential between phases (presence of galvanic cells [18]). Pit density tended to increase when the eutectic was at a higher proportion.

Immerging the sample in the agitated solution (Fig. 6a) generated a stronger attack when compared to the application by drop (Figs. $6 \mathrm{~b}$ and $6 \mathrm{c}$ ). The eutectic region was over-etched when the primary aluminum started to be etched. Application of a droplet may change the reaction kinetics. On the other hand, more pits were produced when the reagent was applied by drop several times $\left(2894 / \mathrm{mm}^{2}\right.$ after 10 seconds single step procedure [b], and $6513 / \mathrm{mm}^{2}$ after two steps procedure with 5 seconds etch at each step[c]). This fact was due to the formation of hydrogen gas bubbles. These gas bubbles can remain on the surface due to the surface tension effect and limit the attainable homogeneity of etching [15]. When the solution was applied twice, the surface cleaning between steps eliminated these bubbles and improved the etching in the subsequent step.

\subsection{DIC analysis}

By analyzing a set of pictures before the test with the DIC software, a subset size of $81 \times 81$ pixels was saved in order to cover at least 3 pits and limit the smoothing effects. The full-field strain was measured for a set of deformed images, and the elastic modulus was calculated by the linear regression method. The elastic modulus obtained by DIC was compliant to standard test results, as shown in Figure 7. This good correlation validated the subset size and the following localized strain calculations.

The analysis of two sets of 30 stationary pictures enabled

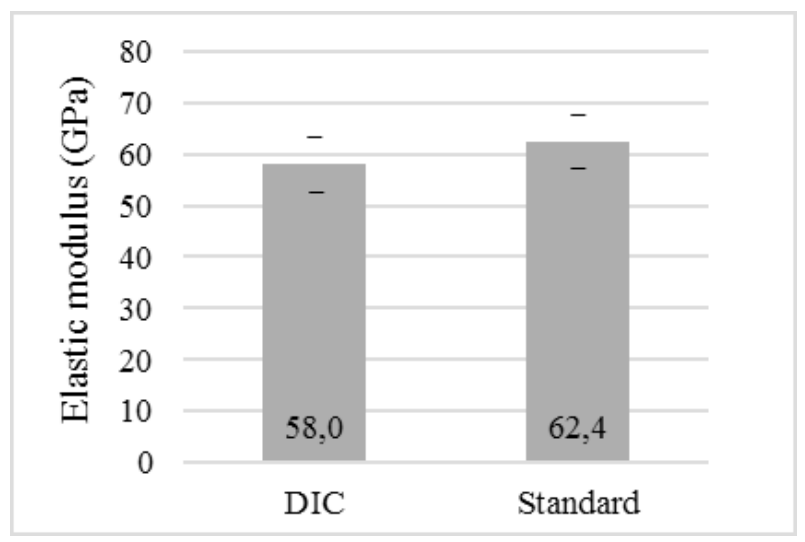

Fig. 7. Comparison between elastic modulus calculated by DIC and standard tensile test, with the regression method

a displacement spatial resolution of $44.3 \pm 5.7 \times 10^{-2} \mu \mathrm{m}$ and a strain spatial resolution of $93.5 \pm 2.3 \times 10^{-6}$. These values are higher than those obtained in the work on cast iron [3]. The reduction of pattern size gave improved spatial resolution and allowed local strain measurements in the different phases or regions. However, the main issue with the developed etching procedure was that, even under the optimized condition, there was still an inhomogeneous spatial distribution. This made the display of strain gradients somewhatuncertain with a certain degree of smoothing. For a steep-strain gradient in a strain fields, smoothing can lead to erroneous results [19]. At a subset size of $81 \times 81$ pixels, local distortions and deviations in the speckle pattern (size and distribution) were considered to be the major contributions to the measurement errors [20]. A consequence of this was that the strain amplitude and gradients between phases may be affected. Moreover, $\alpha$-Aluminum particles generally had a diameter smaller than $100 \mu \mathrm{m}$ (Fig. 5a) and [14]). Therefore, with the strain spatial resolution value obtained, it was difficult to measure the local strains of $\alpha$-Aluminum separately from the eutectic neighbor regions due to the lack of resolution. This issue was verified with Figure 8, where strain values are difficult to relate to microstruture. However, by comparing the strain measured on the line with the full-field strain, Figure 8 highlights the existence of a strain variation around the global strain. The highest values seem to be measured in $\alpha$-Al particles. The $\alpha$-Aluminum is certainly softer and more ductile than the eutectic region. Even if the pattern needs to be enhanced to achive even-higher spatial resolution, it is possible to get a reasonable idea about the relative strain distribution between phases, even if the absolute number is correct.

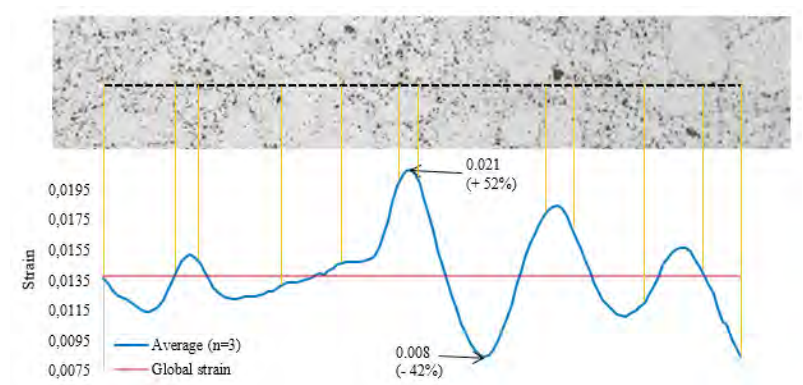

Fig. 8. Evaluation of local strain $(\mathrm{m} / \mathrm{m})$ according to line selected - average of three pictures

\section{CONCLUSION}

Several reagent's concentration, temperatures, and implementation procedures were tested to pit etch a semisolid cast aluminum alloy to enable the measurement of local strain patterns using DIC. It was observed that each reagent had its effect in pit production. Moreover, an issue appeared with the shape of the sample. Results were definitively different according to the type of sample used (standard flat or tensile). A solution containing $70 \%$ phosphoric acid, $5 \%$ acetic acid, 5\% nitric acid, 10\% hydrochloric acid, and $10 \%$ water was prepared at $70^{\circ} \mathrm{C}$ and applied on the sample surfaces by a drop in two steps for 5 seconds each (Fig. 3). The 
pattern produced was not homogeneous and still needs to be improved; however, this procedure allowed for pitting corrosion on both the $\alpha$-Aluminum and eutectic regions of the cast semi-solid A356 aluminum. In-situ tensile tests were performed during the recording of the microstructure. A good fit was found between the elastic modulus calculated by DIC and the standard tests. From this good correlation, strain measurements were performed by DIC; the results showed a local strain variation around the full-field strain calculated. This brought to light the local deformation of this material during loading. However, the spatial resolution has to be enhanced in order to measure local strains more precisely and relate them with microstructure heterogeneities. For this, improved solutions should be developed; for instance, heating the sample instead of the solution could change the reactivity of the material. Then, measuring the pitting corrosion potential of each phase could help understanding the phenomena that occur during pit etching.

\section{Acknowledgments}

The authors wish to acknowledge the Knowledge Foundation for funding this work under the CompCAST project (Dnr. 20100280) and the ERASMUS exchange program for student exchange.

\section{REFERENCES}

[1] Campillo M., Baile M.T., Menargues S. \& Forn A. (2010). The effect of injection conditions on the structural integrity of the components produced by semi-solid Rheocasting. International Journal Material Forming, 3, no. suppl. 1, 751-754.

[2] Menargues S., Martín E., Baile M.T. \& Picas J.A. (2015). New short T6 heat treatments for aluminium silicon alloys obtained by semisolid forming. Materials Science and Engineering $A, 621,236-242$.

[3] Kasvayee K.A., Elmquist L., Jarfors A.E.W. \& Ghassemali E. (2014). Development of a pattern making method for strain Measurement on microstructural level in ferritic cast iron. Processing and Fabrication of Advanced Materials, 23, 1121-1135.
[4] Schreier H., Orteu J.J. \& Sutton M.A. (2009). Image correlation for shape, motion and deformation measurements: Basic concepts, theory and applications, Springer US.

[5] Kasvayee K.A., Ghassemali E. \& Jarfors A.E.W. (2015). Micro-crack initiation in high-silicon cast iron during tension loading. In The Minerals, Metals \& Materials Society (Ed.) TMS2015: 144 $4^{\text {th }}$ Annual Meeting \& Exhibition, Supplemental proceedings, Springer International Publishing 947-953.

[6] Kasvayee K.A., Ghassemali E., Salomonsson K. \& Jarfors A.E.W. (2015). Microstructural strain localization and crack evolution in ductile iron. JTH research report.

[7] A356.0 aluminium casting alloy (7Si-0.3Mg) (2012). Hadleigh Castings, 3-4.

[8] Kaufman J.G. \& Rooy E.L. (2004). Aluminum Alloy Castings: Properties, Processes, and Applications. ASM International.

[9] Linder J. (2012). Alcoholate corrosion of aluminium in ethanol blends - the effects of water content, surface treatments, temperature, time and pressure. Master thesis. KTH Royal Institute of Technology, Stockholm, Sweden.

[10] Ghali E. (2000). Aluminum and aluminum alloys. In R. Winston Revie (Ed.) Uhlig's Corrosion Handbook, Second Edition, New York: John Wiley \& Sons, Inc. 677-690.

[11] Lee J., Kim J., Kim J., Lee J., Chung H. \& Tak Y. (2009). Effects of pretreatment on the aluminium etch pit formation. Corrosion Science, 51(7), 1501-1505.

[12] Beck T.R. (1988) Size distribution of etch pits in aluminum. Electrochimica. Acta, 33(10), 1321-1327.

[13] Bellenger F. (2002). Etude et contrôle de la corrosion feuilletante des alliages d'aluminium 2024 et 7449 par bruit électrochimique et émission acoustique. Analyse microstructurale et caractérisation de l'endommagement. Lyon: L'Institut National des Sciences Appliquées de Lyon.

[14] Granath O., Wessén M. \& Cao H. (2008) Determining effect of slurry process parameters on semisolid A356 alloy microstructures produced by RheoMetal process. International Journal of Cast Metals Research, 21(5), 349-356.

[15] Wolf S. \& Tauber R.N. (1986). Silicon Processing for the VLSI Era, Volume 1 - Process Technology. Latticce Press, 531-535.

[16] Reu P. (2014). Speckles and their relationship to the digital camera.Experimental Technique, 38(4), 1-2.

[17] MatchID 2D. Retrieved from: http://matchidmbc.be/2D.html (accesed 15.12.2017).

[18] Arrabal R., Mingo B., Pardo A., Mohedano M., Matykina E. \& Rodríguez I. (2013). Pitting corrosion of rheocast A356 aluminium alloy in 3.5wt.\% NaCl solution. Corrosion Science, $73,342-355$.

[19] Lecompte D., Smits A., Bossuyt S., Sol H., Vantomme J., Van Hemelrijck D. \& Habraken A.M. (2006) Quality assessment of speckle patterns for digital image correlation. Optics and Lasers in Engineering, 44(11), 1132-1145.

[20] Hung P-C. \& Voloshin A.S. (2003). In-plane strain measurement by digital image correlation. Journal of the Brazilian Society of Mechanical Sciences and Engineering, 25(3), 215-221. 